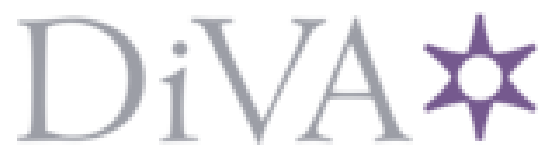

http://www.diva-portal.org

\title{
Postprint
}

This is the accepted version of a paper published in Journal of Applied Physics. This paper has been peer-reviewed but does not include the final publisher proof-corrections or journal pagination.

Citation for the original published paper (version of record):

Kubart, T., Aijaz, A. (2017)

Evolution of sputtering target surface composition in reactive high power impulse magnetron sputtering.

Journal of Applied Physics, 121(17): 171903

https://doi.org/10.1063/1.4977814

Access to the published version may require subscription.

N.B. When citing this work, cite the original published paper.

Permanent link to this version:

http://urn.kb.se/resolve?urn=urn:nbn:se:uu:diva-316657 


\section{Evolution of sputtering target surface composition in reactive High Power Impulse Magnetron Sputtering}

T. Kubart, ${ }^{1, a)}$ A. Aijaz ${ }^{1)}$

${ }^{1}$ Solid State Electronics, The Ångström Laboratory, Uppsala University, P.O. Box 534, SE-75121, Sweden

The interaction between pulsed plasmas and surfaces undergoing chemical changes complicates physics of reactive High Power Impulse Magnetron Sputtering (HiPIMS). In this study, we determine the dynamics of formation and removal of a compound on titanium surface from the evolution of discharge characteristics in argon atmosphere with nitrogen and oxygen. We show that the time response of a reactive process is dominated by surface processes. Thickness of the compound layer is several $\mathrm{nm}$ and its removal by sputtering requires ion fluence in the order of $10^{16} \mathrm{~cm}^{-2}$, much larger than the ion fluence in a single HiPIMS pulse. Formation of the nitride or oxide layer is significantly slower in HiPIMS than in dc sputtering under identical conditions. Further, we explain very high discharge currents in HiPIMS by the formation of truly stoichiometric compound during the discharge off-time. The compound has very high secondary electron emission coefficient and leads to a large increase in the discharge current upon target poisoning.

\section{Introduction}

High Power Impulse Magnetron Sputtering (HiPIMS) is a variant of magnetron sputtering which offers significant improvements over the standard technique. The discharge is operated in a pulsed manner at low duty cycle and low frequency to achieve a very high plasma density. Thanks to the high instantaneous peak powers, plasma densities about two orders of magnitude higher than in standard sputtering are achieved during the pulse on-time. ${ }^{1}$ This leads to significantly enhanced ionization of the sputtered species with beneficial impact on the properties of the deposited coatings. ${ }^{2,3}$ A major advantage of the HiPIMS technique is its compatibility with standard magnetron sputtering equipment, only the power supply has to be replaced.

In reactive sputtering, a wide range of compound films may be prepared from metal targets with a reactive gas added to the argon working atmosphere. Chemical reactions between the sputtered metal and the reactive gas lead to the deposition of a compound. The ion assistance in reactive HiPIMS has been successfully employed for deposition of various compound thin films with improved properties. For instance to control the phase formation in $\mathrm{TiAIN}^{4}$ or $\mathrm{TiO}_{2}{ }^{5}$; reducing the growth temperature of thermochromic $\mathrm{VO}_{2} ;{ }^{6,7}$ controlling the crystal orientation of $\alpha-\mathrm{Fe}_{2} \mathrm{O}_{3} ;{ }^{8}$ or increasing the value of refractive index of $\mathrm{Ta}_{2} \mathrm{O}_{5} .{ }^{9}$

HiPIMS may also make the reactive deposition process more stable by reducing the process hysteresis. The hysteresis is a significant technological issue caused by the formation of compound on the sputtering target surface. An abrupt transition between the so-called metal mode with a clean metallic target, and poisoned or compound mode with the sputtering target surface covered by a layer of compound, leads to a steep reduction of the deposition rate. ${ }^{10}$ The transition is accompanied by a pronounced change in the plasma impedance and leads to instabilities. Although the beneficial impact of HiPIMS on the process hysteresis has been demonstrated in several cases, ${ }^{9,11}$ the exact mechanism of the hysteresis reduction is still disputed.

In general, control of the growth conditions is essential for deposition of high quality materials. HiPIMS provides additional flexibility in the energy and ionization of the film forming species. Optimization of the growth for various materials, however, requires an understanding of the relation between external process parameters, such as the discharge power density or discharge

\footnotetext{
a) Author to whom correspondence should be addressed. Electronic mail: Tomas.Kubart@angstrom.uu.se
} 
pressure, and the resulting energy and composition of the particle flux to the substrate. There is a clear need for a model of the reactive HiPIMS in order to reduce the trial-and-error in process development.

Several models have been presented to describe the plasma physics in nonreactive HiPIMS, which can predict the ionization as well as deposition rate as a function of operating conditions. ${ }^{12-14}$ There is, however, no model available for reactive HiPIMS yet. Only Kozak and VIcek ${ }^{15}$ presented a parametric model for reactive HiPIMS extending the classical approach pioneered by Berg. ${ }^{10}$ This is due to the complexity of the problem; in addition to the plasma region, such a model has to account for the plasma-surface interactions. In the presence of a reactive gas, the surfaces undergo chemical changes and a reliable description of the surface processes is challenging. Moreover, the dynamics of the surface processes has to be correctly reproduced because of the pulsed nature of HiPIMS discharges.

A good test for such a comprehensive model is the hysteresis behaviour. Despite its importance, the exact mechanism of hysteresis reduction in reactive HiPIMS is still disputed and several competing mechanisms have been discussed. For example, it has been proposed that fast cleaning of the target surface causes the observed increased deposition rate in reactive HiPIMS. ${ }^{11,16}$ Others reported formation of a very thick surface oxide layer on the order of hundreds of $\mathrm{nm}$ in reactive HiPIMS of $\mathrm{Ti}^{17,18}$ The thickness of an oxide layer formed by ion implantation is proportional to the ion range which is only few $\mathrm{nm}$ at the energy determined by the discharge voltage. ${ }^{19}$ Much thicker oxide layers would obviously have a pronounced impact on the sputtering process.

Surface composition also influences the composition of the deposition flux. Measured energies of reactive gas ions in HiPIMS discharges suggest that the ions originate from the target surface rather than from the gas phase. ${ }^{20}$ It is therefore important to determine the partial sputtering yield of the reactive gas from the target surface to evaluate the contribution of sputtering to the total reactive gas ion flux.

In this work, the dynamics of compound formation and removal on the sputtering target surface in reactive HiPIMS is studied. The main goal of this study is to identify the dominating physical mechanisms and provide a description of the ion-surface interaction suitable for comprehensive plasma models of HiPIMS discharges. Because in-situ compositional measurements of sputtering targets are virtually impossible, discharge characteristics are used as an indicator of the surface composition. For the sake of an insightful analysis, comparison with the pulsed dc Magnetron Sputtering (pdcMS) study is also made.

\section{Experimental Details}

The evolution of the surface composition was determined from the discharge characteristics, namely the value of the peak current at a constant discharge voltage. This is possible because magnetron discharges are low-pressure cold cathode discharges maintained by secondary electrons emitted from the sputtering target. The plasma impedance is therefore very sensitive to the ioninduced secondary electron emission coefficient (SEEC). ${ }^{21}$ Since the SEEC is determined by the surface composition as well as the energy and type of the impinging ion, the discharge current at a constant voltage provides a direct indication of the changes in the surface composition. ${ }^{22}$ In reactive sputtering, this fact is demonstrated by a large change in the plasma impedance when the sputtering target surface is poisoned, i.e. covered by a layer of compound. In our experiments, this difference is utilized to distinguish between the metal and compound mode of the sputtering. Following the 
transition from metal to compound mode and back enabled us to determine the time, or ion fluence, required to form a layer of compound on the target surface or to remove it.

Dynamic response of a reactive sputtering process is determined not only by the sputtering target but also influenced by the reactive gas distribution in a vacuum system. Times in the order of seconds are required to achieve a stable reactive gas partial pressure after a change in the gas supply rate. ${ }^{23}$ Upon target poisoning, long transition times are caused by the large gettering capacity of surfaces covered by unreacted metal. When reducing the supply of reactive gas, removal of the reactive gas from the system is the main factor. In order to exclude the effects, which are significantly slower than the period of pulsing in HiPIMS, the experiments were operated in a sequential manner providing sufficient time to reach a stable gas composition without any discharge.

The experimental procedure illustrated in Figure 1 is inspired by the approach of Depla et al. employed for dc reactive sputtering. ${ }^{22}$ The target was first sputtered in pure argon corresponding to the metal mode operation, Figure 1a). Then, reactive gas was introduced without discharge. When a stable operating pressure was reached, the initially metallic target surface was poisoned Figure $1 \mathrm{~b}$ ). After reaching compound mode discharge as confirmed by stable discharge current Figure 1c), reactive gas was removed from the chamber. Target cleaning took place during the last discharge period, Figure 1d). The discharge was operated in constant voltage mode with a burst of 900 and 4000 pulses in the HiPIMS and pulsed dc mode (pdcMS), respectively, and the whole burst was recorded to follow the transient behaviour. In order to identify suitable discharge voltage values for the experiments, discharge characteristics in a continuous constant power operation were determined first.

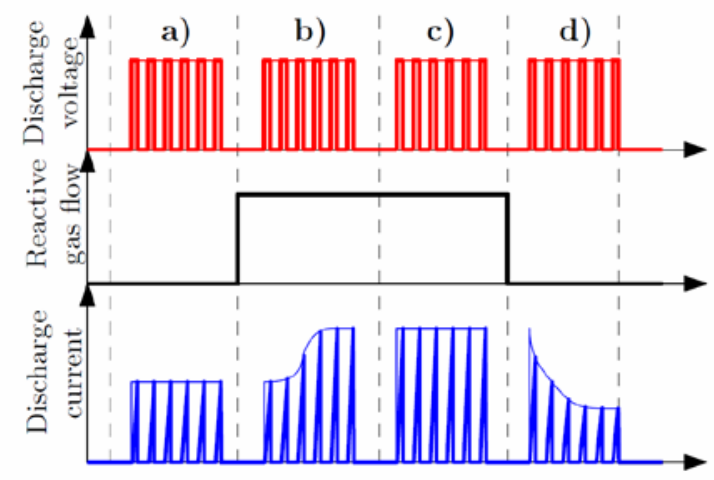

Figure 1: The experimental sequence used in this work consisted of four pulse bursts: a) Metal mode of sputtering with the target sputtered in pure Ar to produce a clean metal surface; b) Target poisoning in $\mathrm{Ar}+\mathrm{O}_{2} / \mathrm{N}_{2}$ atmosphere; c) Compound mode sputtering in $\mathrm{Ar}+\mathrm{O}_{2} / \mathrm{N}_{2}$ with the target covered by a layer of compound; d) Target cleaning in pure Ar to remove the surface compound. Constant discharge voltage was used and the peak current during each burst was recorded. A dwell between the bursts allowed for the reactive gas partial pressure stabilization.

\section{A. Experimental system}

Experiments were performed in Lesker CMS-18 ultra-high vacuum deposition system with a base pressure below $10^{-5}$ Pa pumped by a cryopump (CTI CryoTorr 8 ). Geometry of the deposition system is shown in Figure 2. The system was equipped with four magnetron sputtering sources tilted with an angle of $16^{\circ}$ in co-sputtering configuration. Target-to-substrate distance was approximately $180 \mathrm{~mm}$. Titanium (99.995\%) target with a thickness and diameter of 6 and $100 \mathrm{~mm}$, respectively, was used. All experiments were carried out with an Ar flow of $40 \mathrm{sccm}$ and various flows of oxygen 
and nitrogen gas (99.9995\%) at a constant total pressure of $0.53 \mathrm{~Pa}$ (4 mtorr) maintained by varying the pumping speed. The gases were introduced into the chamber by means of a gas distribution ring around the substrate holder. A second magnetron equipped with aluminium target was running at a low pulsed dc power during the experiments to improve the ignition of the HiPIMS discharge. The discharge voltage of this magnetron was also monitored and served as an indication of the reactive gas partial pressure. This aluminium target was operated behind a closed shutter.

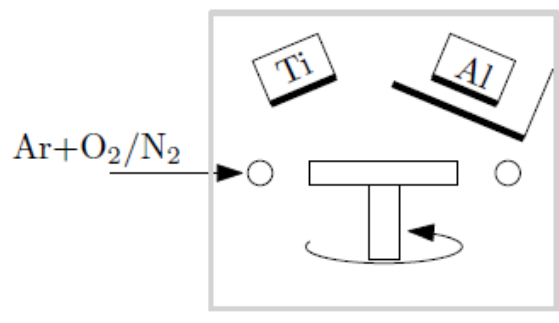

Figure 2: Schematic sketch of the experimental geometry. Two magnetron sources in a co-sputtering geometry were used and the process gases were introduced through a distribution ring around the substrate holder.

The sputtering power was supplied by a SIPP 1000 pulsing unit fed by an Advanced Energy Pinnacle dc generator. Both the target current and voltage were monitored using current and voltage transducers, and recorded in Agilent Infiniium (DSO9064A) digital oscilloscope, which was also used to calculate the instantaneous power delivered to the discharge. The pulsing unit was controlled by an arbitrary function generator (Tektronix AFG 3200) enabling both continuous and pulsed operation with an arbitrary pulse train.

In all the experiments, a pulse-on-time of $100 \mu$ s was used. used For HiPIMS, the period of pulsing was $2 \mathrm{~ms}$, which corresponded to a duty cycle of $5 \%$. The same on-time was used for dc-like experiments with the period of pulsing reduced to $200 \mu \mathrm{s}$ (pdcMS). In this case, the duty cycle was $50 \%$ with correspondingly lower power density. The average power in both, HiPIMS and pdcMS mode was kept comparable.

\section{B. Model of surface sputtering}

Removal of the compound layer from the target surface was simulated by a binary collision approximation (BCA) to provide a further insight into the surface sputtering. The simulations of the sputtering were performed using code TRIDYN ${ }^{24}$ TRIDYN is a BCA code similar to the popular program SRIM. Unlike the static SRIM, TRIDYN takes into account changes in the surface composition caused by sputtering and can therefore describe the evolution of the surface composition. Details of the model are provided elsewhere, ${ }^{25}$ and only a brief summary is provided here. In the simulations, a titanium target covered by $2 \mathrm{~nm}$ thick layer of $\mathrm{TiN}_{\text {or }} \mathrm{TiO}_{2}$ was bombarded by a flux of argon atoms with the energy of $550 \mathrm{eV}$ corresponding to the discharge voltage. Each simulation was run with a total ion fluence of $10^{17} \mathrm{~cm}^{-2}$ simulating $10^{6}$ particle histories and using a cut-off energy of $3 \mathrm{eV}$. The concentration of reactive gas in the target was limited to the stoichiometric limit (67 at.\% for $\mathrm{TiO}_{2}$ and 50 at.\% for TiN) with the excess atoms diffusing towards the surface. ${ }^{26}$ Recoils below the relocation threshold energy (19, 30 and $30 \mathrm{eV}$ for Ti, $\mathrm{N}$ and $\mathrm{O}$, respectively) were bound and the bulk binding energies were set to zero. Surface binding energy dependent on the actual surface composition was used for titanium with the value of $4.89 \mathrm{eV}$ for metallic Ti and Ti in TiN, and $10 \mathrm{eV}$ for $\mathrm{Ti}$ in $\mathrm{TiO}_{2}{ }^{25} \mathrm{~A}$ constant value of 5 and $10 \mathrm{eV}$ was used for the surface binding energy of $\mathrm{N}$ and $\mathrm{O}$. 


\section{Results}

\section{A. Discharge current and voltage waveforms in continuous sputtering}

The discharge current and voltage waveforms for discharges continuously operated at a constant power of $700 \mathrm{~W}$ in pdcMS and HiPIMS are shown in Figure 3 and Figure 4. In the case of pdcMS, the discharge voltage was $456 \mathrm{~V}$ in metal model (pure argon), and it increased to $553 \mathrm{~V}$ and $510 \mathrm{~V}$ in oxide (10 sccm of oxygen) and nitride mode (15 sccm of nitrogen), respectively. Our results are in good agreement with results published for reactive sputtering of titanium. ${ }^{27}$ An increase in the discharge voltage is expected as a result of the decreased ion induced SEEC of oxidized and nitrided titanium. ${ }^{22}$

There is a striking difference in the discharge current behaviour in reactive HiPIMS when either oxygen or nitrogen was introduced, Figure 4 . The discharge current increased significantly with both gases. Further increase in the reactive gas flow did not yield any significant change indicating completely poisoned target, not shown. In addition, the shape of the discharge current was different and no saturation of the current was observed in contrast to the metal mode. Note the difference in the discharge current magnitude between HiPIMS and pdcMS operation that is related to the 10 times lower duty cycle in the HiPIMS experiments. Similar discharge behaviour in reactive HiPIMS has been reported for a range of materials such as titanium, ${ }^{28,29}$ ruthenium, ${ }^{30}$ hafnium, ${ }^{31}$ aluminium, ${ }^{32}$ or tungsten. ${ }^{33}$

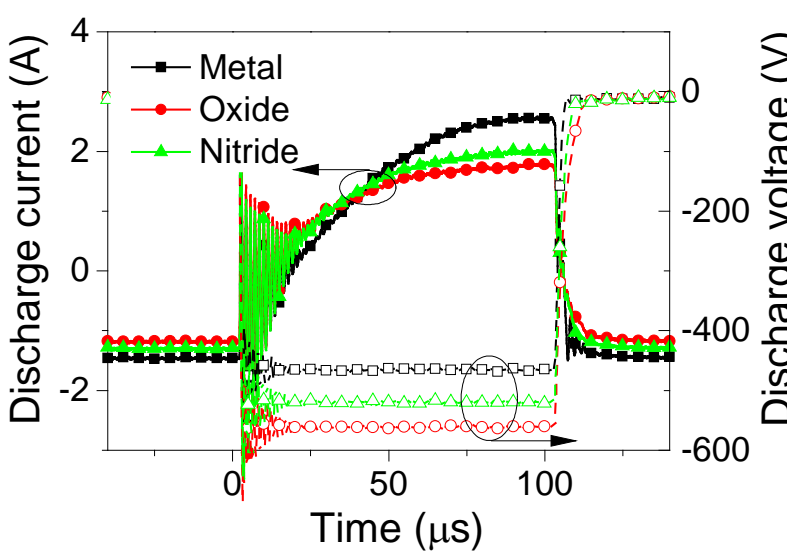

Figure 3: Discharge current (solid) and voltage (dashed, open symbols) pdcMS waveforms in metal, oxide and nitride mode for continuously operated discharges. The average power was 700 W, the pulse on-time 100 us, and the period of pulsing was 200 us.

The continuous sputtering was used to determine discharge voltages and reactive gas flows suitable for the sequential experiments. The values used in the following sections, where the discharges were operated at a constant voltage, are summarized in Table 1.

Table 1: Discharge voltages and reactive gas flows used in the sequential experiments.

\begin{tabular}{lcccc}
\hline \hline & Target & $\begin{array}{c}\text { Reactive } \\
\text { gas }\end{array}$ & $\begin{array}{c}\text { Gas flow } \\
\text { (sccm) }\end{array}$ & $\begin{array}{c}\text { Voltage } \\
\text { (V) }\end{array}$ \\
\hline \multirow{2}{*}{ HiPIMS } & \multirow{2}{*}{$\mathrm{Ti}$} & $\mathrm{O}_{2}$ & 10 & 620 \\
& & $\mathrm{~N}_{2}$ & 15 & 620 \\
\multirow{2}{*}{ pdcMS } & $\mathrm{Ti}$ & $\mathrm{O}_{2}$ & 10 & 520 \\
& & $\mathrm{~N}_{2}$ & 15 & 450 \\
\hline \hline
\end{tabular}

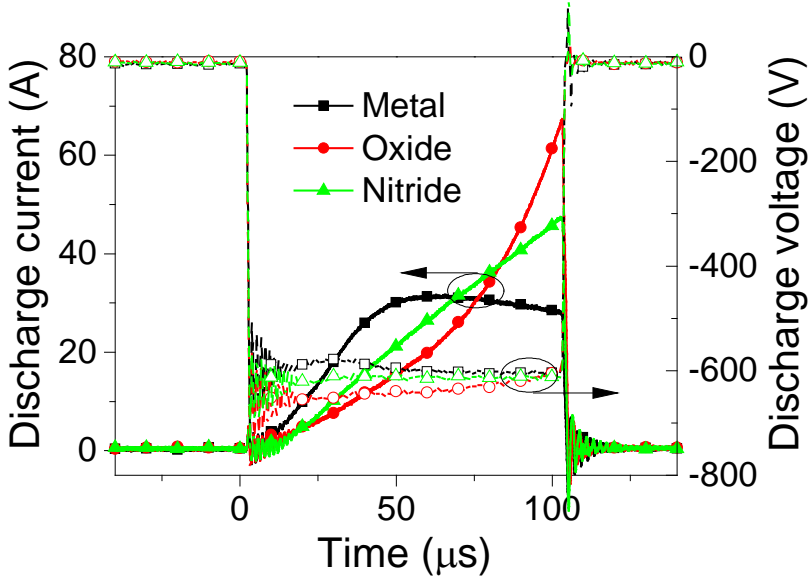

Figure 4: Discharge current (solid) and voltage (dashed, open symbols) HiPIMS waveforms in metal, oxide and nitride mode for continuously operated discharges. The average power was kept at $700 \mathrm{~W}$, and the pulse on-time was $100 \mu \mathrm{s}$ with the period of pulsing of $2 \mathrm{~ms}$. 


\section{B. Target cleaning and poisoning in HiPIMS}

The peak current at a constant voltage is used as an indicator of the target surface composition. Sequential HiPIMS experiments were carried out following the routine from Figure 1. Results with oxygen and nitrogen are shown in Figure 5 and Figure 6, respectively. There is a pronounced difference in the peak current between metal and compound mode and the time required for removal of the surface compound can be estimated from the peak current evolution during cleaning. ${ }^{34}$ The cleaning times are compared to simulations of the surface composition in section $D$. The compound formation time is determined in the same way from the poisoning curves.

For oxygen, very fast target poisoning was observed, Figure $5 b)$. This is in contrast to the poisoning in nitrogen, Figure $6 \mathrm{~b})$, where a single burst was not enough to reach the nitride mode. More than $4 \mathrm{~s}$ of sputtering was necessary to cover the surface with nitride. After the poisoning, a steady state was achieved as confirmed by the stable peak current, Figure 5c) and Figure 6c). Interestingly, very high initial discharge current occurred in the oxygen experiments, Figure 5 b)-d). This was the case even when cleaning the oxidised target in pure argon, Figure $5 \mathrm{~d}$ ), and sputtering a clean metal target during oxidation, Figure 5 b). High discharge current at the onset of cleaning was also observed for the nitride covered target, Figure 6d).

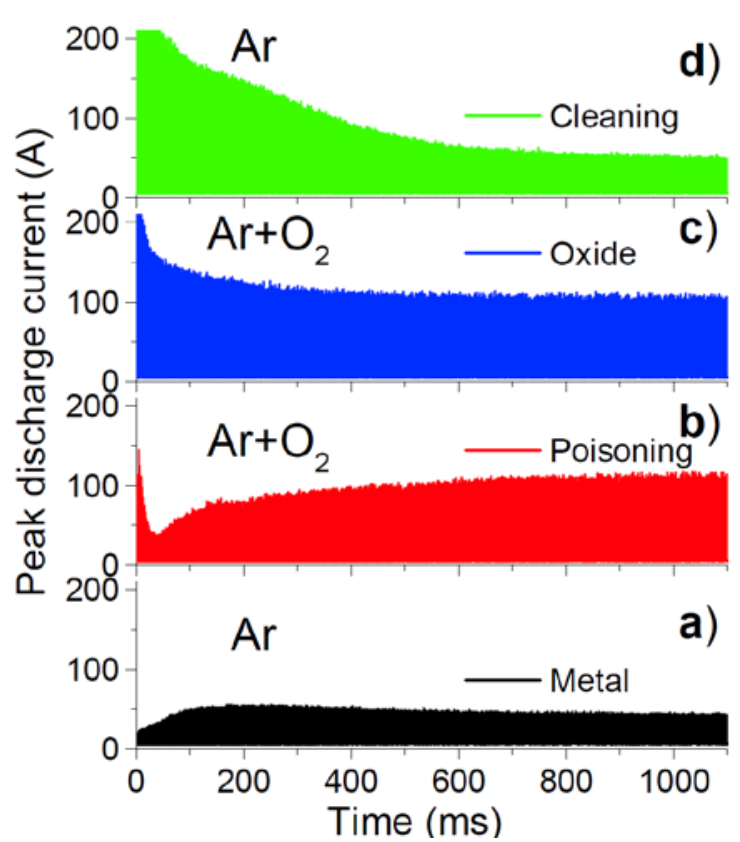

Figure 5: The peak current evolution during a burst of HiPIMS pulses at a constant discharge voltage of $620 \mathrm{~V}$ in reactive sputtering with oxygen.

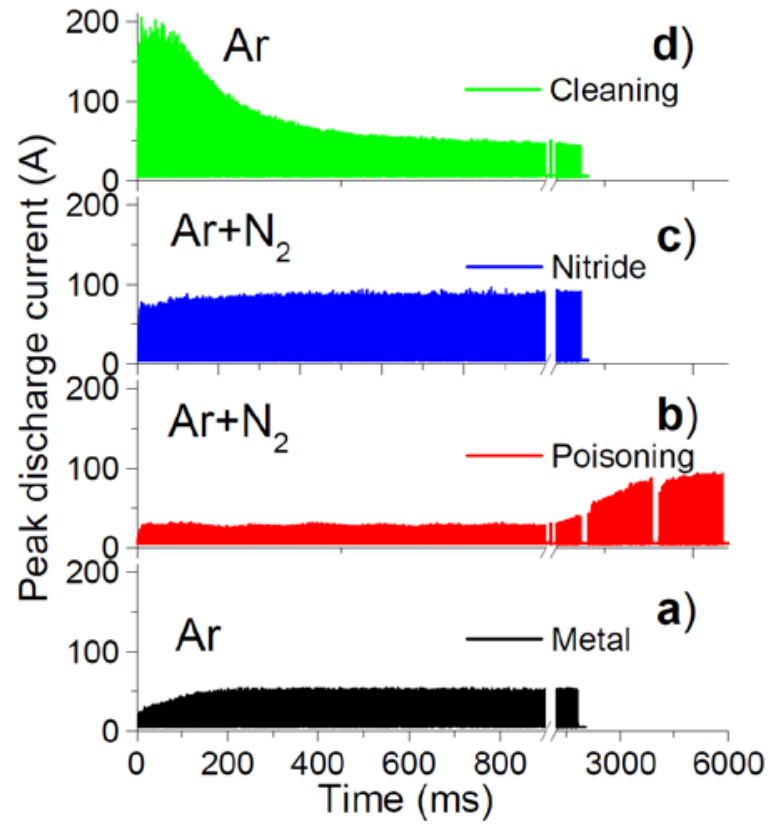

Figure 6: The peak current evolution during a burst of HiPIMS pulses at a constant discharge voltage of $620 \mathrm{~V}$ in reactive sputtering with nitrogen.

Poisoned targets were sputter-cleaned in a pure argon atmosphere. The discharge current evolution during cleaning experiments is shown in more detail in Figure 7 where the discharge current during cleaning and in the metal mode is compared. Cleaning times determined from the exponential decay of the discharge current were 685 and $430 \mathrm{~ms}$ for the oxide and the nitride, respectively. 

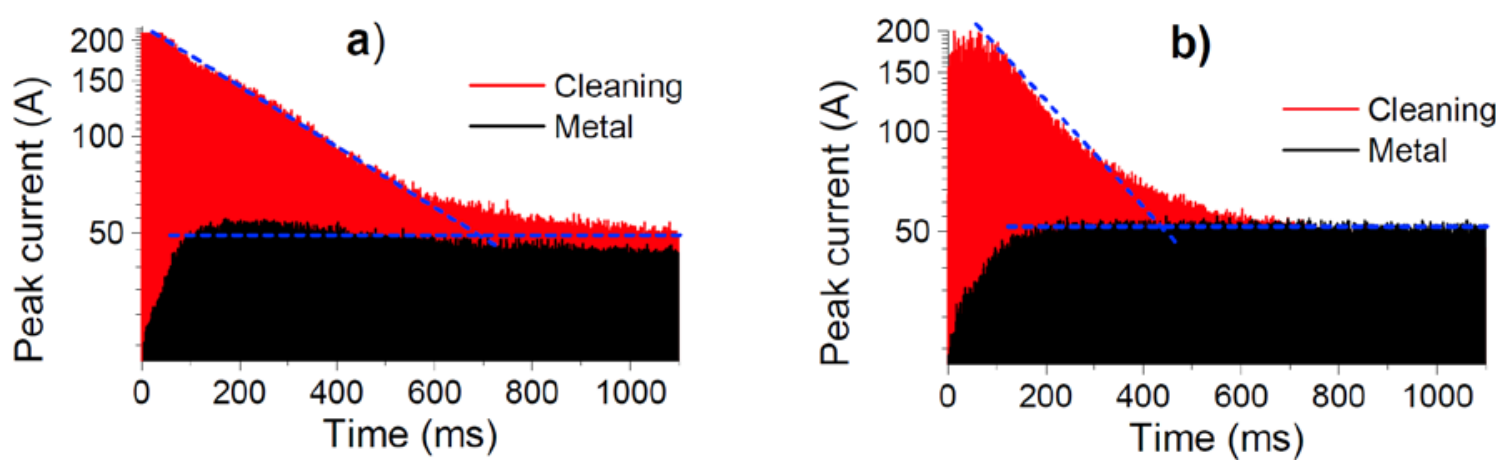

Figure 7: The peak current during HiPIMS cleaning of an oxidised (a) and nitrided (b) titanium target. The discharge current in metal mode is shown for comparison.

Assuming a triangular shape of the current waveform and taking an average peak current $\bar{I}$ during cleaning, the ion fluence required for compound removal in HiPIMS may be determined as

$$
\Phi=\frac{t_{o n} N \bar{I} / 2}{q_{e} A_{t}}
$$

where $t_{\text {on }}$ is the on-time, $N$ number of pulses required for cleaning, $q_{e}$ electron charge and $A_{t}$ the target area, $78 \mathrm{~cm}^{2}$. Since the period of HiPIMS pulsing was $2 \mathrm{~ms}$, cleaning of an oxidised target required 343 pulses while about 215 pulses were required to remove the nitride. Taking $\bar{I}=150 \mathrm{~A}$ for the oxide and $100 \mathrm{~A}$ for nitride, the ion fluence for HiPIMS cleaning can be estimated as $2.1 \cdot 10^{17} \mathrm{~cm}^{-2}$ and $8.6 \cdot 10^{16} \mathrm{~cm}^{-2}$. Here, a uniform distribution of the ion current over the whole target surface is assumed.

\section{Target cleaning and poisoning in pdcMS}

HiPIMS results are compared to pdcMS in order to identify behaviour characteristic for the HiPIMS process. The poisoning-cleaning experiments for pdcMS conditions were performed by reducing the period of pulsing to $200 \mu$ s and adjusting the discharge voltage. The discharge voltage was set to 520 and $450 \mathrm{~V}$ for experiments with oxygen and nitrogen, all other conditions were kept the same as in HiPIMS. The results are shown in Figure 8. In pdcMS case, higher discharge current was observed in the metal mode in agreement with the waveforms from Figure 3. Target poisoning, not shown, was very fast in both nitrogen and oxygen. Most interesting is the fast formation of nitride where, in stark contrast to the HiPIMS, no significant delay occurred.
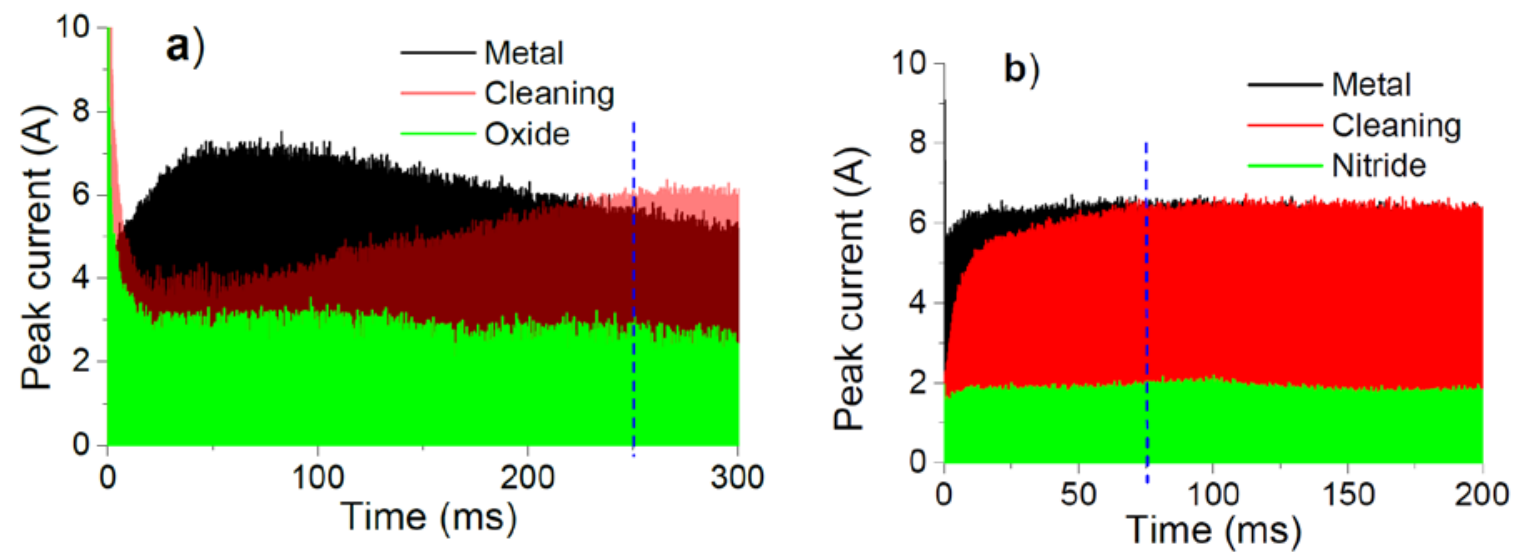

Figure 8: Pulsed DC cleaning of an oxidised (a) and nitrided (b) titanium target and corresponding behaviour in the metal and compound mode. 
Compound removal was faster in pdcMS than in HiPIMS. The cleaning time was about $250 \mathrm{~ms}$ for oxide and $75 \mathrm{~ms}$ for nitride. This corresponds to 1250 and 375 pulses, respectively. At 5 A peak current and approximately rectangular current waveforms, the resulting ion fluence for cleaning was $5.0 \cdot 10^{16} \mathrm{~cm}^{-2}$ and $1.5 \cdot 10^{16} \mathrm{~cm}^{-2}$. Both fluences are significantly lower than the HiPIMS values.

\section{Modelling of the cleaning process}

TRIDYN simulations were carried out for a surface covered by $2 \mathrm{~nm}$ of stoichiometric compound, $\mathrm{TiN}_{\text {or }} \mathrm{TiO}_{2}$, bombarded by $550 \mathrm{eV}$ Ar. It should be noted that the model is very sensitive to the values of input parameters and the results should be considered only qualitative.

Simulated partial sputtering yields of titanium and reactive gas are shown in Figure 9 for both compounds. There is a good qualitative agreement with the pdcMS results. The initial value of the reactive gas partial sputtering yield from both compounds is comparable which is surprising at first, given the much lower erosion rate of $\mathrm{TiO}_{2}$ as compared to TiN. Ranjan et al. determined the sputtering yield of titanium by Ar bombardment at $500 \mathrm{eV}$ to 0.69 from Ti and 0.42 from TiN. ${ }^{35}$ Much lower titanium sputtering yield is typically observed for $\mathrm{TiO}_{2}$, about 10 times lower than the yield from a clean surface..$^{25}$ The simulated yields for titanium are in good agreement with these values and the partial yield of titanium from $\mathrm{TiO}_{2}$ is only about 0.05 . Still, partial sputtering yield of 0.3 and 0.35 is predicted for oxygen and nitrogen, respectively.



Figure 9: TRIDYN simulations of Ar sputtering of a Ti target covered by $2 \mathrm{~nm}$ of $\mathrm{TiN}$ and $\mathrm{TiO}_{2}$. Partial sputtering yields of $\mathrm{Ti}$ and the corresponding reactive gas are shown as a function of fluence.

Initially, the sputtering yields are stable. This corresponds to the sputter erosion of the compound layer. Reactive gas is sputtered off the surface or recoil implanted deeper into the target. Then, the sputtering yield of reactive gas is steadily decreasing as its concentration is being reduced. The total ion dose for sputter removal of the $2 \mathrm{~nm}$ thick compound layer, defined here as completed when the partial sputtering yield of titanium has reached 0.5 atoms/ion, was $8.5 \cdot 10^{16} \mathrm{~cm}^{-2}$ for oxide and $2.7 \cdot 10^{16} \mathrm{~cm}^{-2}$ for nitride. Although there is a fraction of the reactive gas still left in the target, its partial sputtering yield is low. The simulations also reproduce the faster removal of nitride which is caused by higher sputtering yield and lower nitrogen concentration in the stoichiometric compound. 


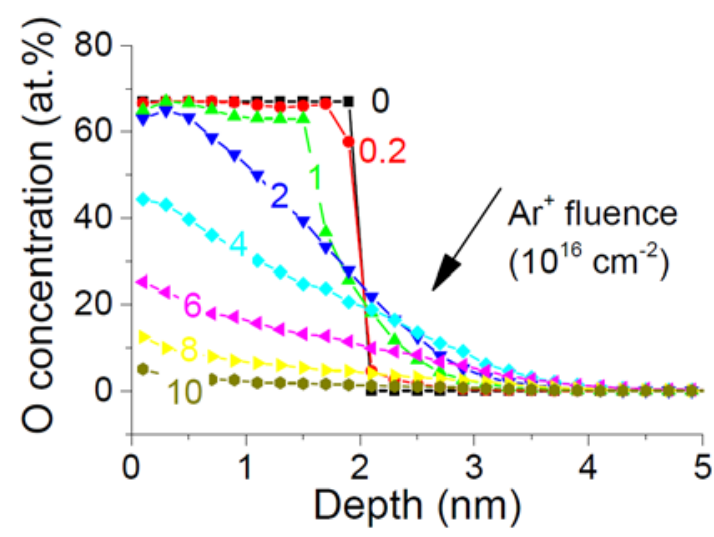

Figure 10: Simulated oxygen compositional depth profiles. The ion fluence in one HiPIMS pulse with oxygen is about $6 \cdot 10^{14} \mathrm{~cm}^{-2}$ and the value of $0.2 \cdot 10^{16} \mathrm{~cm}^{-2}$ thus corresponds to 3 HiPIMS pulses.

Figure 10 shows simulated concentration of oxygen for increasing ion fluences. Clearly, the surface composition changes very slowly and until $2 \cdot 10^{16} \mathrm{~cm}^{-2}$ (corresponding to about 33 HiPIMS pulses) the surface oxygen concentration is rather stable.

\section{Discussion}

Here, we discuss three aspects of the HiPIMS process: i) high initial discharge currents in experiments with oxygen, ii) cleaning times required to remove the compound from poisoned surfaces, and iii) slow target poisoning, and show the effect of surface composition on the discharage characteristics.

Characteristics of magnetron discharges are governed by the SEEC of the target surface. In general, higher SEEC is expected for oxides than metals, i.e. the discharge voltage typically decreases upon target oxidation. The increase in the SEEC is caused by kinetic emission which is significant for oxides already at low ion energies in the range of hundreds of eV. ${ }^{36}$ Depla et al. analysed the effect of surface oxidation on SEEC and proposed a mechanism explaining the peculiar behaviour of titanium: Since $\mathrm{TiO}_{2}$ is very sensitive to preferential sputtering, a wide range of oxides with lower stoichiometry is formed under ion bombardment on the surface. The suboxides reduce the effective SEEC due to the reduction of escape probability of electrons. ${ }^{22}$ This results in a reduced SEEC of the oxidised target and lower discharge current in pdcMS discharges in agreement with the data in Figure 3.

We propose that the high initial current observed in the HiPIMS experiments with oxygen, Figure $5 \mathrm{~b}$-d), may be explained by the different stoichiometry of the surfaces. Due to the pulsed nature of the HiPIMS process, there is no ion bombardment of the target between the discharge pulses and no sputtering occurs. A thin layer of stoichiometric $\mathrm{TiO}_{2}$, therefore, forms by chemisorption due to the high sticking coefficient of thermal oxygen on titanium between the pulses. ${ }^{37}$ Such a thin oxide appears already during the dwell prior to the target poisoning as demonstrated by the high initial current, Figure $5 \mathrm{~b}$ ). Since the oxide is formed only by chemisorption, it is quickly removed at the beginning of poisoning both by sputtering and knock-on implantation ${ }^{19}$, as evidenced by the dip in the discharge current after about $50 \mathrm{~ms}$, Figure $5 \mathrm{~b}$ ). The surface stoichiometry has dominating effect on the discharge characteristics. Even in the absence of oxygen, the initial discharge current is very high during cleaning of an oxidized surface, Figure $5 \mathrm{~d}$ ). The peak current in argon is actually even higher that in the $\mathrm{Ar}+\mathrm{O}_{2}$ mixture possibly due to the higher ionization energy of argon (15.76 eV) than that of oxygen (13.62 eV). 
The discharge characteristics in HiPIMS are influenced by a number of parameters and at this point, it is impossible to to model the behaviour. There is, however, a clear connection between the surface composition and the peak current. Our conclusions are supported by recent results from ion beam experiments reported by Corbella et al. ${ }^{38}$ In their experiments, the authors studied argon ion induced SEEC from titanium oxide surface and showed that the values of SEEC are higher than for a pure titanium surface, in contrast to the results from dc magnetron sputtering. This may be a result of lower ion current density in their experiments and thus a shift towards higer stoichiometry. In the case of HiPIMS, therefore, even higher SEEC may occur.

Assuming a relation between the peak current and surface composition, the peak current during cleaning expriments, Figure 7, would indicate a continuous removal of the surface compound. In order to validate this assumption, the cleaning behaviour is compared to the results from a the simulation discussed in III.D. The ion fluence in cleaning experiments was determined from the number of pulses and the average discharge current according to (1). The results summarized in Table 2 show that the ion fluence required for surface cleaning was rather high, corresponding to several hundreds of HiPIMS pulses. The results are in good agreement with the simulations considering that a constant value of $2 \mathrm{~nm}$ was used for the compound thickness in all cases. Varying the compound thickess may improve the agreement, given the simplifications used in the model, however, the accuracy of such an approach is only limited.

Table 2: Summary of the ion fluence values and times required for cleaning in experiments with nitrogen and oxygen. Values from simulations with $2 \mathrm{~nm}$ thick compound layers are also shown.

\begin{tabular}{llllll}
\hline \hline Reactive & \multicolumn{2}{l}{ lon fluence } & $\left(10^{16} \mathrm{~cm}^{-2}\right)$ & & \multicolumn{2}{l}{ Cleaning time $(\mathrm{ms})$} \\
gas & HiPIMS & pdcMS & Model & HiPIMS & pdcMS \\
\hline $\mathrm{O}$ & 21 & 5 & 8.5 & 685 & 250 \\
$\mathrm{~N}$ & 8.6 & 1.5 & 2.7 & 430 & 75 \\
\hline \hline
\end{tabular}

Although the ion fluence for cleaning was higher in HiPIMS than in pdcMS, our experiments did not show any indication of extremely thick oxide layers reported earlier. ${ }^{17,18}$ It should be noted, however, that because of the sequential operation of the discharge, target heating was negligible and no thermal diffusion of oxygen occurred. Higher values in HiPIMS may be caused by thicker compound layers because of higher ion energy and thus deeper reactive gas ion implantation. Moreover, the simulations did not take into account the possibility of back-attraction of ionised species which may be substantial in HiPIMS. ${ }^{39}$

In summary, our results show that once the surface is covered with a compound, there is a very small change in the surface stoichiometry during a single pulse. This is further evidenced in Figure 10, where the surface oxygen concentration is shown as function of ion fluence and number of pulses. The surface concentration stays very high because the surface is replenished by the implanted oxygen. The continuous decrease in the peak current is in a good agreement with the simulated concentration of oxygen in the surface layer.

Slower target poisoning in HiPIMS than in dcMS is counterintuitive. Due to the high plasma density, higher degree of dissociation of the reactive gas and hence more reactive atmosphere is expected in HiPIMS than in dcMS. ${ }^{40}$ Moreover, the deposition rate in HiPIMS is generally lower for the same discharge power, therefore less reactive gas is incorporated into the deposited film and the partial pressure of the reactive gas is higher in HiPIMS at the same discharge power and reactive gas supply. ${ }^{41}$ Faster formation of a compound would there be expected for the HiPIMS process. In spite 
of that, target poisoning was much faster in all pdcMS experiments. This may be a result of reduced effective reactive gas flux to the surface due to gas rarefaction ${ }^{42}$ or preferential sputtering of the reactive gas due to the higher energy of impinging ions in HiPIMS. ${ }^{43}$ The exact mechanism cannot be deduced from present experiments and requires further investigations.

Higher compound formation rate in oxygen as compared to nitrogen can be explained by the higher oxygen affinity to titanium compared to nitrogen. The enthalpy of formation of $\mathrm{TiO}_{2}$ and $\mathrm{TiN}$ is -944 and $-388 \mathrm{~kJ} / \mathrm{mol}^{44}$ and the sticking coefficient of nitrogen on titanium is lower than that of oxygen. While the oxygen sticking coefficient is close to unity, Mao et al. reported values as low as 0.1 even for atomic nitrogen on titanium. ${ }^{45}$ Both facts results in the slower nitride formation.

\section{Conclusions}

Our experiments show that the dynamics in reactive HiPIMS is dominated by surface processes. The compound formation on a sputtering target surface and its removal by sputtering require much longer time, or ion fluence, than a single typical HiPIMS pulse can provide. The compound formation rate is significantly inhibited in HiPIMS as compared to the dc mode of operation. Thickness of the oxide layer is in $\mathrm{nm}$ range and no indications of very thick surface layers were observed in HiPIMS.

High discharge current in the compound mode of reactive HiPIMS is explained by the formation of a stoichiometric oxide on the sputtering target. It is argued that a truly stoichiometric oxide is formed in HiPIMS due to the absence of sputtering between discharge pulses. It is further proposed that the SEEC of this oxide is very high and close to the values expected for stoichiometric oxides although the exact mechanism remains to be studied further.

\section{Acknowledgements}

Dr Stephanos Konstantinidis and Dr Joakim Andersson are gratefully acknowledged for helpful discussion and valuable comments. The work was financially supported by the Swedish Foundation for Strategic Research.

\section{References}

J. T. Gudmundsson, N. Brenning, D. Lundin, and U. Helmersson, Journal of Vacuum Science \& Technology A 30, 030801 (2012).

2 D. Lundin and K. Sarakinos, Journal of Materials Research 27, 780 (2012).

$3 \quad$ A. Anders, Surface \& Coatings Technology 257, 308 (2014).

$4 \quad$ G. Greczynski, J. Lu, J. Jensen, S. Bolz, W. Kolker, C. Schiffers, O. Lemmer, J. E. Greene, and L. Hultman, Surface \& Coatings Technology 257, 15 (2014).

$5 \quad$ S. Konstantinidis, J. P. Dauchot, and A. Hecq, Thin Solid Films 515, 1182 (2006).

6 J. P. Fortier, B. Baloukas, O. Zabeida, J. E. Klemberg-Sapieha, and L. Martinu, Solar Energy Materials and Solar Cells 125, 291 (2014). A. Aijaz, Y.-X. Ji, J. Montero, G. A. Niklasson, C. G. Granqvist, and T. Kubart, Solar Energy Materials and Solar Cells 149, 137 (2016). Olejnicek, M. Cada, I. Gregora, and R. Zboril, Acs Nano 9, 7113 (2015). M. Hala, R. Vernhes, O. Zabeida, J. E. Klemberg-Sapieha, and L. Martinu, Journal of Applied Physics 116, 213302 (2014).

$10 \quad$ S. Berg and T. Nyberg, Thin Solid Films 476, 215 (2005).

11 E. Wallin and U. Helmersson, Thin Solid Films 516, 6398 (2008).

12 C. Q. Huo, D. Lundin, M. A. Raadu, A. Anders, J. T. Gudmundsson, and N. Brenning, Plasma Sources Science \& Technology 23, 025017 (2014). 
T. Kozak, J. Vlcek, and S. Kos, Journal of Physics D-Applied Physics 46, 105203 (2013).

C. Huo, M. A. Raadu, D. Lundin, J. T. Gudmundsson, A. Anders, and N. Brenning, Plasma Sources Science \& Technology 21, 045004 (2012).

T. Kozak and J. Vlcek, Journal of Physics D: Applied Physics 49, 055202 (2016).

M. Hala, J. Capek, O. Zabeida, J. E. Klemberg-Sapieha, and L. Martinu, Journal of Physics DApplied Physics 45, 055204 (2012).

M. Audronis, G. Abrasonis, F. Munnik, R. Heller, P. Chapon, and V. Bellido-Gonzalez, Journal of Physics D: Applied Physics 45, 375203 (2012).

M. Palmucci, PhD Thesis Thesis, University of Mons, 2013.

D. Depla and R. De Gryse, Surface \& Coatings Technology 183, 184 (2004).

M. Aiempanakit, A. Aijaz, D. Lundin, U. Helmersson, and T. Kubart, Journal of Applied Physics 113, 133302 (2013).

M. A. Lieberman and A. J. Lichtenberg, Principles of Plasma Discharges and Materials Processing, Vol. 2nd (Willey -Interscience, New Jersey, 2005).

D. Depla, S. Heirwegh, S. Mahieu, J. Haemers, and R. De Gryse, Journal of Applied Physics 101, 013301 (2007).

T. Kubart, O. Kappertz, T. Nyberg, and S. Berg, Thin Solid Films 515, 421 (2006).

W. Moller, W. Eckstein, and J. P. Biersack, Computer Physics Communications 51, 355 (1988).

T. Kubart, T. Nyberg, and S. Berg, Journal of Physics D: Applied Physics 43, 205204 (2010).

W. Moller and D. Guttler, Journal of Applied Physics 102, 094501 (2007).

D. Depla, G. Buyle, J. Haemers, and R. De Gryse, Thin Solid Films 515, 468 (2006).

V. Stranak, M. Quaas, H. Wulff, Z. Hubicka, S. Wrehde, M. Tichy, and R. Hippler, Journal of Physics D-Applied Physics 41, 055202 (2008).

F. Magnus, O. B. Sveinsson, S. Olafsson, and J. T. Gudmundsson, Journal of Applied Physics 110, 083306 (2011).

D. Benzeggouta, M. C. Hugon, and J. Bretagne, Plasma Sources Science \& Technology 18, 045026 (2009).

R. Ganesan, B. J. Murdoch, B. Treverrow, A. E. Ross, I. S. Falconer, A. Kondyurin, D. G. McCulloch, J. G. Partridge, D. R. McKenzie, and M. M. M. Bilek, Plasma Sources Science \& Technology 24, 035015 (2015).

M. A. Moreira, T. Torndahl, I. Katardjiev, and T. Kubart, Journal of Vacuum Science \& Technology A (Vacuum, Surfaces, and Films) 33, 021518 (2015).

M. A. Vyvoda, C. F. Abrams, and D. B. Grave, leee Transactions on Plasma Science 27, 1433 (1999).

R. Schelfhout, K. Strijckmans, F. Boydens, and D. Depla, Applied Surface Science 355, 743 (2015).

R. Ranjan, J. P. Allain, M. R. Hendricks, and D. N. Ruzic, Journal of Vacuum Science \&amp; Technology A 19, 1004 (2001).

A. V. Phelps and Z. L. Petrovic, Plasma Sources Science \& Technology 8, R21 (1999).

M. C. Burrell and N. R. Armstrong, Langmuir 2, 30 (1986).

C. Corbella, A. Marcak, T. de los Arcos, and A. von Keudell, Journal of Physics D-Applied

Physics 49, 16 lt01 (2016).

J. Vlcek and K. Burcalova, Plasma Sources Science \& Technology 19, 065010 (2010).

C. Nouvellon, M. Michiels, J. P. Dauchot, C. Archambeau, F. Laffineur, E. Silberberg, S.

Delvaux, R. Cloots, S. Konstantinidis, and R. Snyders, Surface \& Coatings Technology 206, 3542 (2012).

T. Kubart, M. Aiempanakit, J. Andersson, T. Nyberg, S. Berg, and U. Helmersson, Surface \& Coatings Technology 205, S303 (2011).

M. Palmucci, N. Britun, S. Konstantinidis, and R. Snyders, Journal of Applied Physics 114, 113302 (2013).

M. Aiempanakit, T. Kubart, P. Larsson, K. Sarakinos, J. Jensen, and U. Helmersson, Thin Solid Films 519, 7779 (2011). 
W. M. Haynes, CRC Handbook of Chemistry and Physics, 92nd Edition (Internet Version 2012), Vol. 32, 92nd Edition (Internet Version 2012) ed. (CRC Press/Taylor and Francis, Boca Raton, FL., 2012).

D. Mao, K. Tao, and J. Hopwood, Journal of Vacuum Science \& Technology A 20, 379 (2002). 